\title{
Casticin induces ovarian cancer cell apoptosis by repressing FoxM1 through the activation of FOXO3a
}

\author{
LING JIANG $^{1 *}$, XIAO-CHENG CAO ${ }^{2 *}$, JIAN-GUO CAO ${ }^{2}$, FEI LIU ${ }^{2}$, MEI-FANG QUAN ${ }^{2}$, \\ XI-FENG SHENG ${ }^{2}$ and KAI-QUN REN ${ }^{2}$
}

\author{
${ }^{1}$ Department of Gynaecology and Obstetrics, The People's Hospital of Hunan Province, \\ First Affiliated Hospital of Hunan Normal University, Changsha 410005; ${ }^{2}$ Laboratory of Medicine Engineering, \\ Medical College, Hunan Normal University, Changsha 410013, P.R. China
}

Received October 26, 2012; Accepted January 24, 2013

DOI: $10.3892 / 01.2013 .1258$

\begin{abstract}
Casticin, a polymethoxyflavone, is reported to have anticancer activities. The aim of the present study was to examine the molecular mechanisms by which casticin induces apoptosis in ovarian cancer cells. The human ovarian cancer cell lines SKOV3 and A2780 were cultured in vitro. Various molecular techniques, including histone/DNA enzymelinked immunosorbent assay (ELISA), reverse transcription polymerase chain reaction (RT-PCR), western blot analysis and gene transfection, were used to assess the expression of FOXO3a and forkhead box protein M1 (FoxM1) in casticintreated ovarian cancer cell lines. Casticin-induced apoptotic cell death was accompanied by the activation of transcription factor FOXO3a, with a concomitant decrease in the expression levels of FoxM1 and its downstream target factors, namely survivin and polo-like kinase 1 (PLK1), and an increase in p $27^{\mathrm{KIP} 1}$. A small inhibitory RNA (siRNA) knockout of FoxM1 potentiated casticin-induced apoptosis in ovarian cancer cells. Silencing FOXO3a expression using siRNA increased FoxM1 expression levels and clearly attenuated the induction of apoptosis by casticin treatment. These results show that casticin-induced apoptosis in ovarian cancer may be caused by the activation of FOXO3a, leading to FoxM1 inhibition.
\end{abstract}

\section{Introduction}

Ovarian cancer is the most common cause of cancer-associated mortalities arising from gynecological tumors $(1,2)$. The most common treatment approach for ovarian cancer consists of a combination of surgery and chemotherapy. Over

Correspondence to: Dr Kai-Qun Ren, Laboratory of Medicine Engineering, Medical College, Hunan Normal University, No. 36 Lushan Road, Hunan, Changsha 410013, P.R. China

E-mail: kaiqunren@126.com

*Contributed equally

Key words: ovarian cancer, casticin, FOXO3a, forkhead box protein M1, apoptosis the past three decades, surgical tumor debulking followed by platinum-based chemotherapy has been the standard treatment for advanced ovarian cancer. Although response rates and complete responses in advanced disease after first-line treatment with carboplatin and paclitaxel are $>80 \%$ and $40-60 \%$, respectively, the majority of patients eventually relapse, with a median progression-free survival of 18 months (3). Therefore, there is an urgent demand to test novel drugs for the prevention and treatment of ovarian cancer.

Casticin is one of the main components of the fruit of Vitex rotundifolia $L$. Casticin has been shown to exert an anti-inflammatory effect in vivo (4) and has been widely used in traditional Chinese medicine as an anti-inflammatory drug for thousands of years. Increasing numbers of studies have shown that casticin exhibits anticarcinogenic activity in breast (5), cervical $(6,7)$, lung and colon cancer (8-10) and hepatocellular carcinoma (11), in addition to ovarian cancer (12). It has been proposed that cell cycle arrest and casticin-induced apoptosis may be the possible mechanisms of its anticancer effects. However, the precise underlying mechanisms are not fully elucidated.

The forkhead box protein M1 (FoxM1) belongs to a family of evolutionarily conserved transcriptional regulators that are characterized by the presence of a DNA-binding domain called the forkhead box or winged-helix domain $(13,14)$. Numerous studies have demonstrated the biological significance of FoxM1 in controlling tumor aggressiveness. FoxM1 has been shown to be involved in cell proliferation and apoptosis, which affects the developmental function of several organs $(14,15)$. A number of studies have revealed FoxM1 to be a key cell cycle regulator in the transition from $\mathrm{G} 1$ to $\mathrm{S}$ phase and in the progression to mitosis $(16,17)$. The loss of FoxM1 expression leads to mitotic spindle defects, the mitotic delay of cells and induction of mitotic catastrophe or apoptotic cell death (18-20). FoxM1 has also been shown to regulate the transcription of cell cycle genes essential for G1-S and G2-M progression, including survivin, Cdc25B, cyclin B, cyclin D1, p21 ${ }^{\mathrm{CIP} 1}$ and p27 ${ }^{\mathrm{KIP} 1}$ (21-23). FoxM1 has been shown to bind to the mammalian mitotic kinase polo-like kinase 1 (PLK1), thus acting as a mediator of the PLK1-dependent regulation of cell cycle progression $(24,25)$.

FOXO3a is a member of the FOXO subfamily of forkhead transcription factors. The phosphorylation of FOXO3a 
results in the impairment of its DNA-binding ability and an increased binding affinity for the 14-3-3 proteins. This causes FoxM1 upregulation, which in turn promotes cell proliferation and survival (26-28). By contrast, the dephosphorylation of activated FOXO3a induces cell cycle arrest and apoptosis (29-31). Since casticin promotes cell cycle arrest and apoptosis, it is possible that it exerts these antitumor effects by regulating FOXO3a/FoxM1 expression. However, the intracellular mechanisms by which casticin induces apoptosis in ovarian cancer cells via the regulation of FOXO3a/FoxM1 signaling have never been examined. Hence, the aim of the present study was to examine the molecular mechanisms by which casticin-induced activation of the transcription factor FOXO3a induces apoptosis in ovarian cancer cells. Our results demonstrated that casticin promotes the dephosphorylation of FOXO3a, leading to the inhibition of FoxM1, which ultimately causes ovarian cancer cell apoptosis.

\section{Materials and methods}

Chemicals and antibodies. Casticin was purchased from Chengdu Biopurify Phytochemicals Ltd. (Chengdu, Sichuan, China). It has a molecular weight of 374.3, appears as yellow crystals and has a purity of $98.0 \%$. Casticin was prepared in dimethyl sulfoxide (DMSO) as a $10 \mathrm{mmol} / 1$ stock solution and diluted in Dulbecco's minimum essential medium (DMEM; Invitrogen, Carlsbad, CA, USA) to the indicated concentration before use. Primary antibodies FoxM1, PLK1, p2 $7^{\mathrm{KIP} 1}$, survivin, $\beta$-actin, anti-caspase-3 and anti-poly (ADP-ribose) polymerase (PARP) were purchased from Santa Cruz Biotechnology, Inc. (Santa Cruz, CA, USA). Horseradish peroxidase-conjugated rabbit anti-mouse secondary antibody was also purchased from Santa Cruz Biotechnology, Inc. Mouse monoclonal antibodies against FOXO3a and phospho-FOXO3a-Thr ${ }^{32}$ were purchased from Millipore (Bedford, MA, USA). Lipofectamine ${ }^{\mathrm{TM}} 2000$ was purchased from Invitrogen. Protease inhibitor cocktail and all other chemicals were obtained from Sigma (St. Louis, MO, USA).

Cells and cell culture. The human ovarian cancer cell lines SKOV3 and A2780 were purchased from the China Center for Type Culture Collection (CCTCC; Wuhan, China). The cells were maintained in DMEM supplemented with $10 \%$ fetal bovine serum (FBS), $4 \mathrm{mM}$ glutamine, $100 \mathrm{U} / \mathrm{ml}$ penicillin and $100 \mu \mathrm{g} / \mathrm{ml}$ streptomycin and incubated at $37^{\circ} \mathrm{C}$ in a humidified atmosphere of $5 \% \mathrm{CO}_{2}$.

Histone/DNA enzyme-linked immunosorbent assay (ELISA) for detecting apoptosis. The cell apoptosis ELISA detection kit (Roche Applied Sciences, Penzberg, Germany) was used to detect apoptosis in casticin-treated cells, according to the manufacturer's instructions. Briefly, cells were seeded in 96-well plates at a density of $1 \times 10^{4}$ cells/well for $24 \mathrm{~h}$ and the test agents were added to the culture medium containing $10 \%$ FBS. After $24 \mathrm{~h}$, the cytoplasm of cells in the control (untreated) and treatment (casticin-treated) groups was transferred to streptavidin precoated 96-well plates, which had been previously incubated with a biotinylated histone antibody and peroxidase-tagged mouse anti-human DNA for $2 \mathrm{~h}$ at room temperature. The absorbance was measured at $405 \mathrm{~nm}$ using an ELx800 ELISA plate reader (Bio-Tek, Winchester, VA, USA).

Reverse transcription polymerase chain reaction (RT-PCR). Total RNA was extracted using TRIzol ${ }^{\circledR}$ reagent (Life Technologies, Gaithersburg, MD, USA). The integrity of the RNA was assessed using $2 \%$ agarose gel electrophoresis. RNA $(\sim 2 \mu \mathrm{g})$ was reverse transcribed using the SuperScript ${ }^{\mathrm{TM}}$ First-Strand Synthesis System kit (Invitrogen). cDNAs encoding the FoxM1 and glyceraldehyde-3-phosphate dehydrogenase (GAPDH) genes were amplified by PCR as follows: denaturation at $94^{\circ} \mathrm{C}$ for $30 \mathrm{sec}$, annealing at $63^{\circ} \mathrm{C}$ for $30 \mathrm{sec}$ and elongation at $72^{\circ} \mathrm{C}$ for $45 \mathrm{sec}$. The primer sequences were designed as follows: for FoxM1, the forward primer was 5'-AAC CGCTACTTGACATTGG-3' and the reverse primer was 5'-GCAGTGGCTTCATCTTCC-3'. A GAPDH housekeeping gene was used as the internal control, for which the forward primer was 5'-ACCCAGAAGACTGTGGATGG-3' and the reverse primer was 5'-TGCTGTAGCCAAATTCGTTG-3'. PCR products were analyzed by $2 \%$ agarose gel electrophoresis.

RNA interference. FoxM1, FOXO3a and control small interfering RNA (siRNA) were purchased from Santa Cruz Biotechnology, Inc. Human ovarian cancer SKOV3 and A2780 cells were transfected with FoxM1, FOXO3a and control siRNA using Lipofectamine ${ }^{\mathrm{TM}} 2000$, as described by Wang et al (32). The cells were then collected and processed for western blot analysis and histone/DNA ELISA.

Western blot analysis. Total cell extracts for western blot analysis were obtained, as previously described (33). Cell lysates containing $50 \mu \mathrm{g}$ protein were electrophoresed on a $7.5-12.5 \%$ sodium dodecyl sulfate polyacrylamide gel and blotted onto polyvinylidene difluoride membranes (Millipore). Anti-FoxM1, anti-p27 ${ }^{\mathrm{KIP} 1}$, anti-survivin, anti-FOXO3a, anti-phosphoFOXO3a-Thr ${ }^{32}$, anti-caspase-3 and anti-PARP were used as primary antibodies. The blots were stripped and reprobed with an anti-actin antibody to normalize for differences in protein loading. Changes in the levels of the desired proteins were determined by densitometric scanning of the immunoreactive bands and corrected for $\beta$-actin loading control. Immunoblotting for each protein was performed at least twice using independently prepared lysates to ensure reproducibility of the results.

Statistical analysis. The database was set up with the SPSS 15.0 software package (SPSS Inc., Chicago, IL, USA) for statistical analysis. Data are presented as mean \pm standard deviation (SD). The means of multiple groups were compared using one-way analysis of variance (ANOVA) after the equal check of variance and comparisons between the means were performed using the least significant difference (LSD) method. Statistical comparison was also performed using a two-tailed t-test when appropriate. $\mathrm{P}<0.05$ was considered to indicate a statistically significant difference.

\section{Results}

Effects of casticin on apoptosis in ovarian cancer cells. Based on results from studies that demonstrated the casticin-induced 
A

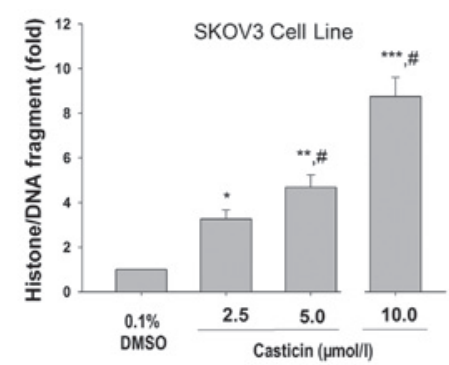

C

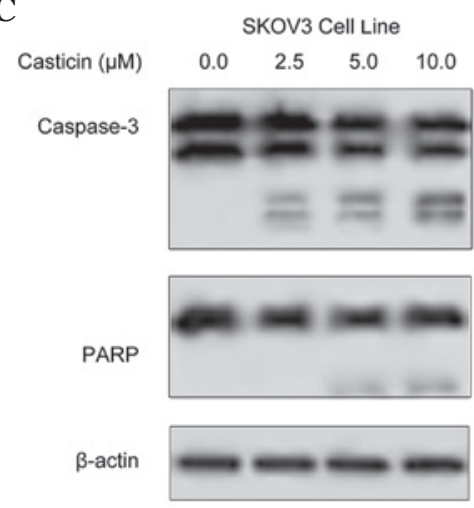

B

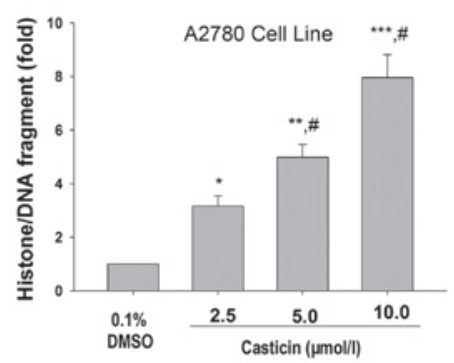

D

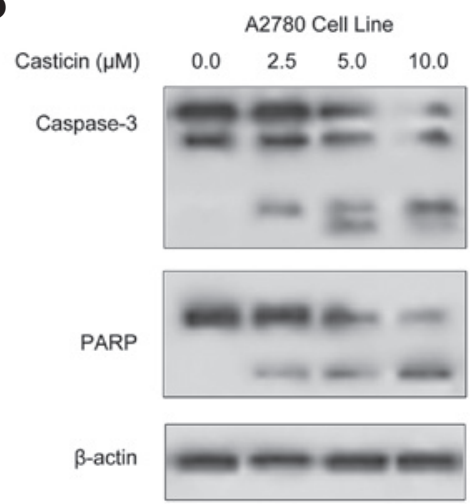

Figure 1. Effects of casticin on apoptosis in ovarian cancer cells. (A and B) SKOV3 and A2780 cells were treated with the indicated concentrations of casticin for $24 \mathrm{~h}$. Histone/DNA fragmentation was determined using ELISA. Data are presented as mean \pm standard deviation $(\mathrm{SD} ; \mathrm{n}=3)$. ${ }^{*} \mathrm{P}<0.05,{ }^{* *} \mathrm{P}<0.01$ and ${ }^{* * * *} \mathrm{P}<0.001$ vs. $0.1 \%$ dimethyl sulfoxide (DMSO); ${ }^{*} \mathrm{P}<0.05$ vs. treatment with $2.5 \mu \mathrm{mol} / 1$ casticin. (C and D) Cell treatment was the same as in $\mathrm{A}$ and $\mathrm{B}$. The expression levels of caspase- 3 and poly (ADP-ribose) polymerase (PARP) were determined by western blot analysis in the total cell lysates and $\beta$-actin was used as the loading control. ELISA, enzyme-linked immunosorbent assay; SD, standard deviation.

growth inhibition of ovarian cancer cells (12) and apoptosis in human cervical cancer $(6,7)$ and hepatocellular carcinoma (11) cells, we first investigated whether casticin was able to induce apoptosis in ovarian cancer cells. Exposing SKOV3 and A2780 cells to $2.5,5.0$ and $10.0 \mu \mathrm{mol} / \mathrm{l}$ casticin for $24 \mathrm{~h}$ significantly induced histone/DNA fragmentation in a concentration-dependent manner (Fig. 1A and B). Additionally, casticin (2.5, 5.0 and $10.0 \mu \mathrm{mol} / \mathrm{l}$ ) activated caspase- 3 and induced the cleavage of its substrate, PARP, in SKOV3 and A2780 cells. Substrate cleavage was indicated by a reduction in the uncleaved forms of caspases and their substrates and/or the appearance of their cleaved forms (Fig. 1C and D). Taken together, these results demonstrated that casticin induces apoptosis in ovarian cancer cells.

Effects of casticin on FoxM1 expression in ovarian cancer cells. Studies have shown that the loss of FoxM1 expression induces apoptosis (19). We investigated whether casticin was able to regulate FoxM1 expression during casticin-induced apoptosis in ovarian cancer cells. The expression of FoxM1 was determined using RT-PCR and western blot analysis. We showed that FoxM1 was overexpressed in SKOV3 (Fig. 2A) and A2780 (Fig. 2B) cell lines. Exposing SKOV3 and A2780 cells to $2.5,5.0$ and $10.0 \mu \mathrm{mol} / \mathrm{l}$ casticin for $24 \mathrm{~h}$ significantly reduced the expression of FoxM1 at the protein level (Fig. 2A and B). Silencing FoxM1 expression using siRNA resulted in the enhanced induction of apoptosis with casticin treatment in SKOV3 (Fig. 2C) and A2780 (Fig. 2D) cell lines.

Effects of casticin on the expression of downstream targets of FoxM1 in ovarian cancer cells. To further confirm the effects of casticin on the functional regulation of FoxM1, we assessed the expression of FoxM1 downstream target genes in SKOV3 and A2780 cells following casticin treatment. FoxM1 is known to have several downstream target genes, including PLK1, survivin and p27 ${ }^{\mathrm{KIP} 1}$ (21,34-36). Western blot analysis revealed that casticin reduced the expression levels of PLK1 and survivin and increased expression of $\mathrm{p} 27^{\mathrm{KIP} 1}$ at the protein level in SKOV3 (Fig. 3A) and A2780 (Fig. 3B) cells. These results provide molecular evidence suggesting that casticin-induced apoptosis in ovarian cancer cells may be mediated via the inactivation of FoxM1.

Effects of casticin on the phosphorylation level of FOXO3a protein in ovarian cancer cells. Since FOXO3a is considered to be an upstream regulator of the FoxM1 transcription factor (29), we investigated the expression of phosphorylated FOXO3a protein in order to explain the mechanism of casticin-dependent FoxM1 inhibition. Western blot analysis showed that treatment with casticin led to a decrease in the FOXO3a phosphorylation level, with a corresponding decrease in FoxM1 expression levels (Fig. 4). These results indicate that casticin-mediated inhibition of FoxM1 expression may be associated with the inhibition of FOXO3a phosphorylation.

Effects of silencing the FOXO3a gene on casticin-mediated apoptosis in ovarian cancer cells. In order to confirm the role of the FOXO3a transcription factor in the cellular response to casticin, we performed gene silencing experiments. SKOV3 and A2780 cells were generated and expression of the FOXO3a protein was attenuated using siRNA technology. In FOXO3a siRNA-transfected cells, the expression levels of FoxM1 
A

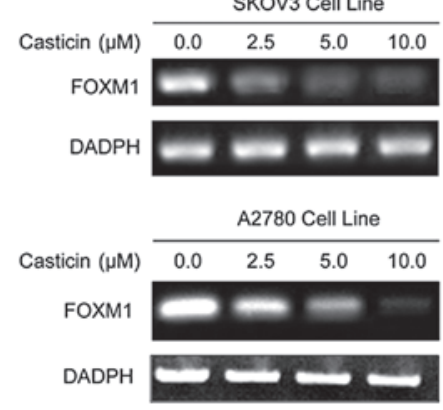

C

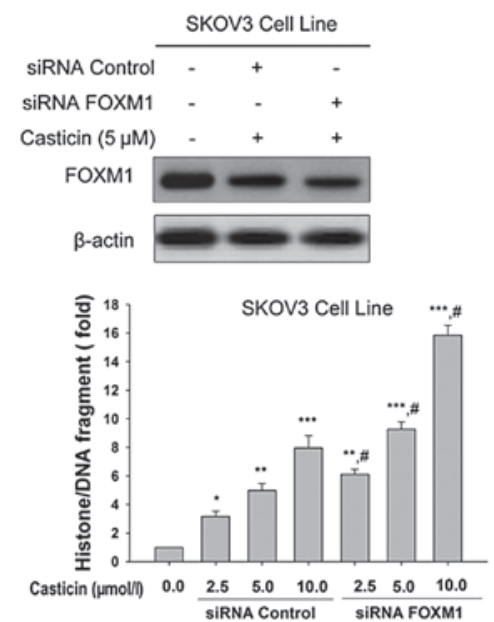

B

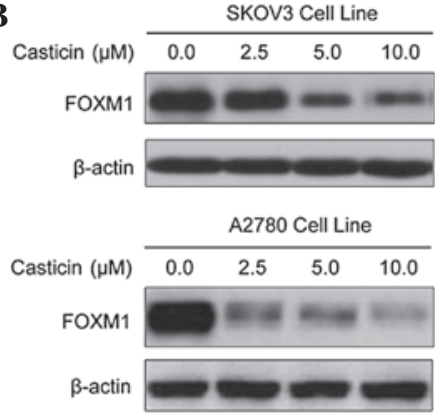

D

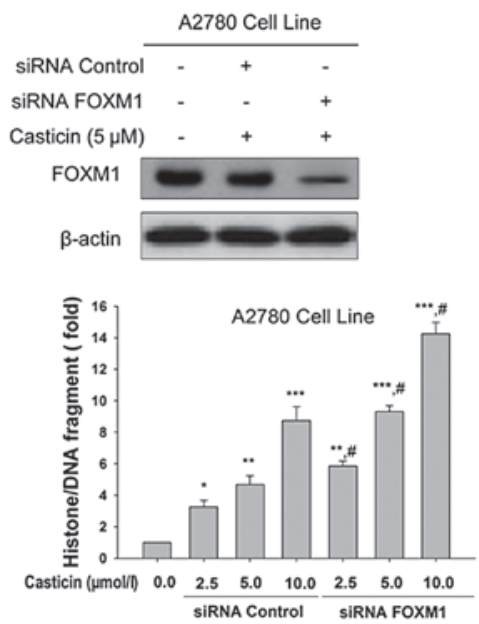

Figure 2. Effects of casticin on the expression of FoxM1 in ovarian cancer cells. (A and B) SKOV3 and A2780 cells were treated with the indicated concentrations of casticin for $24 \mathrm{~h}$. The expression of FoxM1 was determined using (A) RT-PCR and (B) western blot analysis. (C and D) SKOV3 and A2780 cells were transiently transfected with a control non-specific siRNA or FoxM1-specific siRNA for $48 \mathrm{~h}$, followed by treatment with the indicated concentrations of casticin for $24 \mathrm{~h}$. The expression levels of caspase-3 and PARP were determined by western blot analysis in the total cell lysates and $\beta$-actin was used as the loading control. Histone/DNA fragmentation was determined using ELISA. Data are presented as mean \pm SD $(n=3)$. ${ }^{*} \mathrm{P}<0.05$, ${ }^{* *} \mathrm{P}<0.01$ and ${ }^{* * * *} \mathrm{P}<0.001$ vs. $0.1 \%$ DMSO; ${ }^{*} \mathrm{P}<0.05$ vs. cells treated with the same concentration of casticin before being transfected with non-specific siRNA. FoxM1, forkhead box protein M1; RT-PCR, reverse transcription polymerase chain reaction; siRNA, small inhibitory RNA; PARP, poly (ADP-ribose) polymerase; ELISA, enzyme-linked immunosorbent assay; SD, standard deviation; DMSO, dimethyl sulfoxide.

clearly increased (Fig. 5A and B). Additionally, we identified that the knockdown of FOXO3a significantly attenuated casticin-induced apoptosis in ovarian cancer cells (Fig. 5C and D). These findings are consistent with our hypothesis that casticin induces ovarian cancer cell apoptosis by repressing FoxM1 expression through the induction of FOXO3a activity.

\section{Discussion}

In the present study, we showed that casticin induced apoptosis in ovarian cancer cells occurs due to a decrease in the expression levels of FoxM1 and its downstream targets PLK1 and survivin and an increase in $\mathrm{p} 27^{\mathrm{KIP} 1}$, all of which are associated with activation of the FOXO3a transcription factor via dephosphorylation. Additionally, silencing the FOXO3a transcription factor using an siRNA approach blocked casticin-induced downregulation of FoxM1 and inhibited apoptosis. Our results suggest that casticin induces apoptosis in ovarian cancer cells (SKOV3 and A2780) through the regulation of FOXO3a/FoxM1 signaling.

A number of studies have demonstrated overexpression of the FoxM1 gene in human cancer cells and tissues, including in ovarian cancer $(32,33,37,38)$, and emerging evidence suggests that the inactivation of FoxM1 may have important implications in cancer therapy. For example, it may be possible to downregulate FoxM1 expression using specific drugs, such as siomycin $\mathrm{A}$, thiostrepton and the epidermal growth factor receptor (EGFR) inhibitor gefitinib $(27,39,40)$. Wang et al demonstrated that genistein is capable of inhibiting FoxM1 activation in pancreatic cancer cells, leading to cell growth inhibition and the induction of apoptosis (32). In the present study, we investigated the possibility that casticin induces apoptotic cell death and aimed to determine the role of FoxM1 in casticin-dependent ovarian cancer cell apoptosis. Our data showed that casticin elicited a marked effect on apoptosis in ovarian cancer cells, as demonstrated by histone/DNA ELISA results which showed the activation of caspase-3 and cleavage of PARP, accompanied by the downregulation of FoxM1 expression. Our results suggest that FoxM1 is a target of casticin in ovarian cancer cells, as FoxM1 is known to induce oncogenesis and its downregulation causes the inhibition of cell growth (40).

The FOXO3a transcription factor is important in the regulation of the cell cycle and apoptosis $(41,42)$. FOXO3a is regulated by phosphorylation (43). Upon activation of the PI3K/AKT signaling pathway, FOXO proteins undergo AKT-mediated phosphorylation, which promotes their binding to 14-3-3 proteins and facilitates their nuclear export through 
A

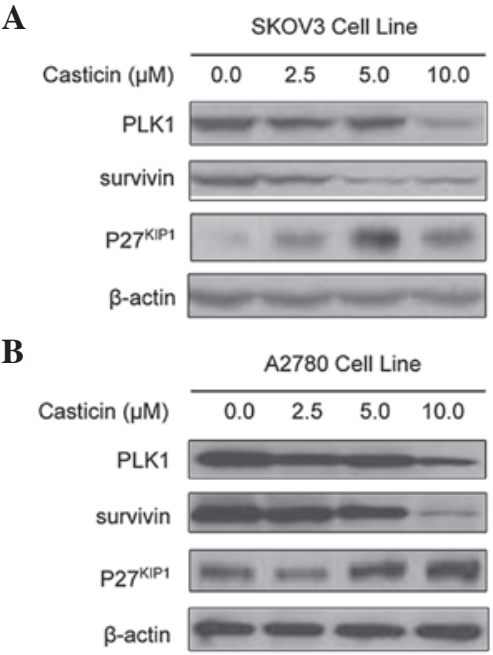

Figure 3. Effects of casticin on the expression of FoxM1 downstream targets in ovarian cancer cells. (A) SKOV3 and (B) A2780 cells were treated with the indicated concentrations of casticin for $24 \mathrm{~h}$. The expression of PLK1, survivin and $\mathrm{p} 27^{\mathrm{KIP} 1}$ was analyzed using western blot analysis. $\beta$-actin was used as a loading control. FoxM1, forkhead box protein M1; PLK1, polo-like kinase 1

A

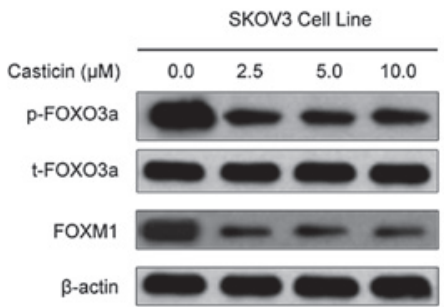

B

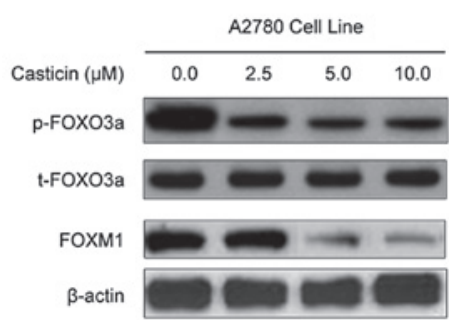

Figure 4. Effects of casticin on the phosphorylation level of FOXO3a protein in ovarian cancer cells. (A) SKOV3 and (B) A2780 cells were treated with the indicated concentrations of casticin for $24 \mathrm{~h}$. The expression of phosphorylated FOXO3a (p-FOXO3a), total FOXO3a (t-FOXO3a) and FoxM1 was analyzed using western blot analysis. $\beta$-actin was used as a loading control. FoxM1, forkhead box protein M1.

chromosome region maintenance 1 (CRM1) and cytoplasmic sequestration. Under conditions of stress or in the absence of growth or survival factors, the PI3K/AKT pathway is inhibited and FOXO3a proteins translocate to the cell nucleus, where they execute their transcriptional functions (40). In the present study, we demonstrated that casticin inhibited FOXO3a phosphorylation. Furthermore, knockdown of FOXO3a by transfection with siRNA blocked the casticin-induced downregulation of FoxM1 expression and inhibited ovarian cancer cell apoptosis. These findings indicate that FOXO3a is a key regulator of casticin-induced apoptosis and FoxM1 expression in ovarian cancer cells.
A

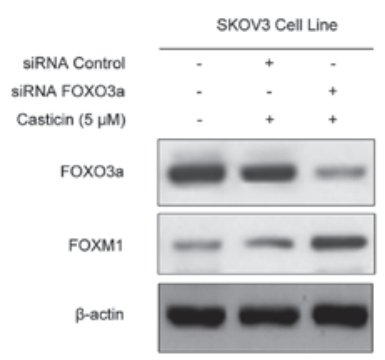

B

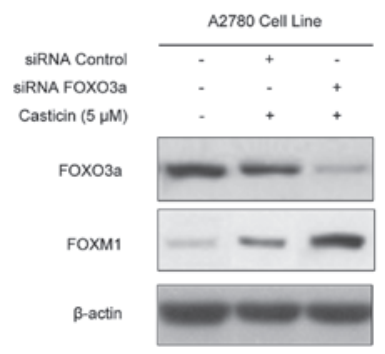

C

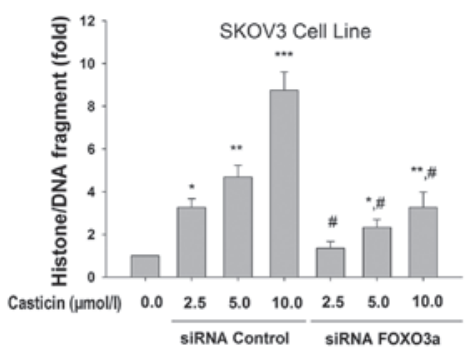

D

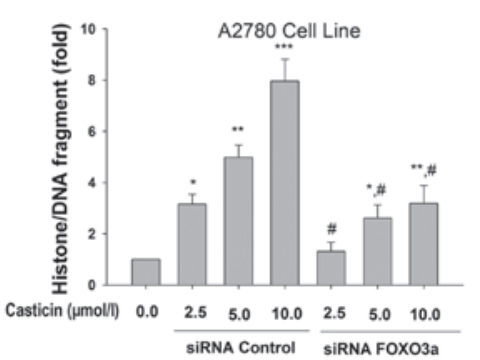

Figure 5. Effects of silencing the FOXO3a gene on casticin-mediated apoptosis in ovarian cancer cells. (A and B) SKOV3 and A2780 cells were transiently transfected with a control non-specific siRNA or FOXO3aspecific siRNA for $48 \mathrm{~h}$, followed by treatment with DMSO or casticin at the indicated concentrations for $24 \mathrm{~h}$. The expression of FOXO3a and FoxM1 was analyzed using western blot analysis. $\beta$-actin was used as a loading control. (C and D) SKOV3 and A2780 cells were transiently transfected with a control non-specific siRNA or FOXO3a-specific siRNA for $48 \mathrm{~h}$, followed by treatment with DMSO or casticin at the indicated concentrations for $24 \mathrm{~h}$. Histone/DNA fragmentation was determined using ELISA. Data are presented as mean $\pm \mathrm{SD}(\mathrm{n}=3) .{ }^{*} \mathrm{P}<0.05,{ }^{* * *} \mathrm{P}<0.01,{ }^{* * *} \mathrm{P}<0.001$ vs. $0.1 \%$ DMSO; ${ }^{\text {" }} \mathrm{P}<0.05$ vs. cells treated with the same concentration of casticin before being transfected with non-specific siRNA. siRNA, small inhibitory RNA; DMSO, dimethyl sulfoxide; ELISA, enzyme-linked immunosorbent assay; FoxM1, forkhead box protein M1; SD, standard deviation.

In summary, our study demonstrated that casticin-induced dephosphorylation of FOXO3a regulates the expression of FoxM1 and its target genes, including survivin, PLK1 and p2 $7^{\mathrm{KIP} 1}$, and this causes apoptosis in ovarian cancer cells. Further studies are required to assess the upstream events leading to FOXO3a phosphorylation and casticin-dependent anticancer effects in ovarian cancer cells. A thorough understanding of the mechanisms and effects of casticin on the cell 
cycle and apoptosis may lead to the identification and development of novel therapeutic molecules for the treatment and prevention of ovarian cancer and other malignant diseases.

\section{Acknowledgements}

This study was supported by the Hunan province Science and Technology Project (No. 2011FJ4144).

\section{References}

1. Lan C, Chenggang W, Yulan B, Xiaohui D, Junhui Z and Xiao W: Aberrant expression of WWOX protein in epithelial ovarian cancer: a clinicopathologic and immunohistochemical study. Int J Gynecol Pathol 31: 125-132, 2012.

2. Vlahovic G, Meadows KL, Uronis HE, et al: A phase I study of bevacizumab, everolimus and panitumumab in advanced solid tumors. Cancer Chemother Pharmacol 70: 95-102, 2012.

3. Hennessy BT, Coleman RL and Markman M: Ovarian cancer. Lancet 374: 1371-1382, 2009 .

4. Lin S, Zhang H, Han T, Wu JZ, Rahman K and Qin LP: In vivo effect of casticin on acute inflammation. Zhong Xi Yi Jie He Xue Bao 5: 573-576, 2007.

5. Li WX, Cui CB, Cai B, Wang HY and Yao XS: Flavonoids from Vitex trifolia $L$. inhibit cell cycle progression at G2/M phase and induce apoptosis in mammalian cancer cells. J Asian Nat Prod Res 7: 615-626, 2005.

6. Chen D, Cao J, Tian L, Liu F and Sheng X: Induction of apoptosis by casticin in cervical cancer cells through reactive oxygen species-mediated mitochondrial signaling pathways. Oncol Rep 26: 1287-1294, 2011.

7. Zeng F, Tian L, Liu F, Cao J, Quan M and Sheng X: Induction of apoptosis by casticin in cervical cancer cells: reactive oxygen species-dependent sustained activation of Jun N-terminal kinase. Acta Biochim Biophys Sin (Shanghai) 44: 442-449, 2012.

8. Díaz F, Chávez D, Lee D, et al: Cytotoxic flavone analogues of vitexicarpin, a constituent of the leaves of Vitex negundo. J Nat Prod 66: 865-867, 2003

9. Haïdara K, Zamir L, Shi QW and Batist G: The flavonoid Casticin has multiple mechanisms of tumor cytotoxicity action. Cancer Lett 242: 180-190, 2006.

10. Imai M, Kikuchi H, Denda T, Ohyama K, Hirobe C and Toyoda H: Cytotoxic effects of flavonoids against a human colon cancer derived cell line, COLO 201: a potential natural anti-cancer substance. Cancer Lett 276: 74-80, 2009.

11. Yang J, Yang Y, Tian L, Sheng XF, Liu F and Cao JG: Casticin-induced apoptosis involves death receptor 5 upregulation in hepatocellular carcinoma cells. World J Gastroenterol 17: 4298-4307, 2011.

12. Hernández MM, Heraso C, Villarreal ML, Vargas-Arispuro I and Aranda E: Biological activities of crude plant extracts from Vitex trifolia L. (Verbenaceae). J Ethnopharmacol 67: 37-44, 1999.

13. Hannenhalli S and Kaestner KH: The evolution of Fox genes and their role in development and disease. Nat Rev Genet 10: 233-240, 2009.

14. Korver W, Roose J and Clevers H: The winged-helix transcription factor Trident is expressed in cycling cells. Nucleic Acids Res 25: 1715-1719, 1997.

15. Kwok JM, Peck B, Monteiro LJ, et al: FOXM1 confers acquired cisplatin resistance in breast cancer cells. Mol Cancer Res 8 24-34, 2010.

16. Bergamaschi A, Christensen BL and Katzenellenbogen BS: Reversal of endocrine resistance in breast cancer: interrelationships among 14-3-3, , FOXM1, and a gene signature associated with mitosis. Breast Cancer Res 13: R70, 2011.

17. Feo F, Frau M and Pascale RM: Interaction of major genes predisposing to hepatocellular carcinoma with genes encoding signal transduction pathways influences tumor phenotype and prognosis. World J Gastroenterol 14: 6601-6615, 2008.

18. Chen W, Yuan K, Tao ZZ and Xiao BK: Deletion of Forkhead Box M1 transcription factor reduces malignancy in laryngeal squamous carcinoma cells. Asian Pac J Cancer Prev 12: 1785-1788, 2011.

19. Halasi M and Gartel AL: Suppression of FOXM1 sensitizes human cancer cells to cell death induced by DNA-damage. PLoS One 7: e31761, 2012.
20. Wonsey DR and Follettie MT: Loss of the forkhead transcription factor FoxM1 causes centrosome amplification and mitotic catastrophe. Cancer Res 65: 5181-5189, 2005.

21. Down CF, Millour J, Lam EW and Watson RJ: Binding of FoxM1 to $\mathrm{G} 2 / \mathrm{M}$ gene promoters is dependent upon B-Myb. Biochim Biophys Acta 1819: 855-862, 2012.

22. Millour J, de Olano N, Horimoto Y, et al: ATM and p53 regulate FOXM1 expression via E2F in breast cancer epirubicin treatment and resistance. Mol Cancer Ther 10: 1046-1058, 2011.

23. Schüller U, Zhao Q, Godinho SA, et al: Forkhead transcription factor FoxM1 regulates mitotic entry and prevents spindle defects in cerebellar granule neuron precursors. Mol Cell Biol 27: 8259-8270, 2007.

24. Fu Z, Malureanu L, Huang J, et al: PLK1-dependent phosphorylation of FoxM1 regulates a transcriptional programme required for mitotic progression. Nat Cell Biol 10: 1076-1082, 2008.

25. Laoukili J, Alvarez-Fernandez M, Stahl M and Medema RH: FoxM1 is degraded at mitotic exit in a Cdh1-dependent manner. Cell Cycle 7: 2720-2726, 2008.

26. Delpuech O, Griffiths B, East P, et al: Induction of Mxi1-SR alpha by FOXO3a contributes to repression of Myc-dependent gene expression. Mol Cell Biol 27: 4917-4930, 2007.

27. McGovern UB, Francis RE, Peck B, et al: Gefitinib (Iressa) represses FOXM1 expression via FOXO3a in breast cancer. Mol Cancer Ther 8: 582-591, 2009.

28. Wilson MS, Brosens JJ, Schwenen HD and Lam EW: FOXO and FOXM1 in cancer: the FOXO-FOXM1 axis shapes the outcome of cancer chemotherapy. Curr Drug Targets 12: 1256-1266, 2011.

29. Fang L, Wang H, Zhou L and Yu D: Akt-FOXO3a signaling axis dysregulation in human oral squamous cell carcinoma and potent efficacy of FOXO3a-targeted gene therapy. Oral Oncol 47: 16-21, 2011.

30. Lam M, Carmichael AR and Griffiths HR: An aqueous extract of Fagonia cretica induces DNA damage, cell cycle arrest and apoptosis in breast cancer cells via FOXO3a and p53 expression. PLoS One 7: e40152, 2012.

31. Yusuf I, Zhu X, Kharas MG, Chen J and Fruman DA: Optimal B-cell proliferation requires phosphoinositide 3-kinasedependent inactivation of FOXO transcription factors. Blood 104: 784-787, 2004.

32. Wang Z, Ahmad A, Banerjee S, et al: FoxM1 is a novel target of a natural agent in pancreatic cancer. Pharm Res 27: 1159-1168, 2010.

33. Ning Y, Li Q, Xiang H, Liu F and Cao J: Apoptosis induced by 7-difluoromethoxyl-5,4'-di-n-octyl genistein via the inactivation of FoxM1 in ovarian cancer cells. Oncol Rep 27: 1857-1864, 2012.

34. Bellelli R, Castellone MD, Garcia-Rostan G, et al: FOXM1 is a molecular determinant of the mitogenic and invasive phenotype of anaplastic thyroid carcinoma. Endocr Relat Cancer 19: 695-710, 2012.

35. Dibb M, Han N, Choudhury J, et al: The FOXM1-PLK1 axis is commonly upregulated in oesophageal adenocarcinoma. Br J Cancer 107: 1766-1775, 2012.

36. Ha SY, Lee CH, Chang HK, et al: Differential expression of forkhead box M1 and its downstream cyclin-dependent kinase inhibitors p27(kip1) and p21(waf1/cip1) in the diagnosis of pulmonary neuroendocrine tumours. Histopathology 60: 731-739, 2012.

37. Cancer Genome Atlas Research Network: Integrated genomic analyses of ovarian carcinoma. Nature 474: 609-615, 2011.

38. Petrovic V, Costa RH, Lau LF, Raychaudhuri P and Tyner AL: FoxM1 regulates growth factor-induced expression of kinase-interacting stathmin (KIS) to promote cell cycle progression. J Biol Chem 283: 453-460, 2008.

39. Kwok JM, Myatt SS, Marson CM, Coombes RC, Constantinidou D and Lam EW: Thiostrepton selectively targets breast cancer cells through inhibition of forkhead box M1 expression. Mol Cancer Ther 7: 2022-2032, 2008.

40. Petrovic V, Costa RH, Lau LF, Raychaudhuri P and Tyner AL: Negative regulation of the oncogenic transcription factor FoxM1 by thiazolidinediones and mithramycin. Cancer Biol Ther 9: 1008-1016, 2010

41. Roy SK, Srivastava RK and Shankar S: Inhibition of PI3K/AKT and MAPK/ERK pathways causes activation of FOXO transcription factor, leading to cell cycle arrest and apoptosis in pancreatic cancer. J Mol Signal 5: 10, 2010.

42. Zanella F, Link W and Carnero A: Understanding FOXO, new views on old transcription factors. Curr Cancer Drug Targets 10: 135-146, 2010.

43. Myatt SS and Lam EW: The emerging roles of forkhead box (Fox) proteins in cancer. Nat Rev Cancer 7: 847-859, 2007. 